\section{Obstetric anaesthesia and MoyaMoya disease}

To the Editor:

We read with interest the letter from Drs. Venkatesh and Taggart ${ }^{1}$ regarding the anaesthetic management of a patient with MoyaMoya disease during Caesarean section. These authors described successful general anaesthesia for this patient and claim it to be the ideal anaesthetic technique. However, there was no discussion of the benefits of regional anaesthesia in this patient. We recently used epidural anaesthesia for a term parturient with MoyaMoya disease undergoing Caesarean section. The outcome was successful and we would like to defend our rationale for using a regional technique in this patient.

The patient with MoyaMoya disease has a compromised cerebral circulation. ${ }^{2}$ There may be an associated intracranial aneurysm, the incidence of which has been estimated to be $14 \%$ in adults. ${ }^{3}$ Identifiable factors precipitating neurological deficit during anaesthesia in these patients include hyperventilation resulting in hypocarbia, ${ }^{4}$ severe hypotension, and subarachnoid haemorrhage due to sudden fluctuation in systemic arterial pressure. ${ }^{5}$

In the obstetric population, central neural block is preferred over general anaesthesia for elective Caesarean section. The risk of maternal aspiration and neonatal drug depression is thereby reduced, and factors that increase cerebral metabolic oxygen consumption, such as the stress response to laryngoscopy and tracheal intubation, are avoided during regional anaesthesia. The hypertensive response to intubation may be detrimental particularly in such cases as MoyaMoya disease with associated intracranial aneurysms, and may increase the risk of intracerebral haemorrhage. Also, during regional anaesthesia, the anaesthetist remains in communication with the patient, and can monitor cerebral function by observing the level of consciousness and the appearance of any abnormal neurological signs.

However, MoyaMoya vessels and other normal collateral vessels in the ischaemic hemisphere are maximally dilated. We agree with the authors that, in these patients, it is especially important to avoid hypotension in order to maintain cerebral blood flow and to prevent further ischaemia. Although epidural anaesthesia may be associated with hypotension, incremental dosing through a catheter and appropriate fluid preloading minimizes this risk. We used epidural anaesthesia in this way and successfully avoided extensive sympathetic blockade and hypotension. Uterine displacement was maintained throughout the procedure. We also catheterized a radial artery for continuous monitoring for hypotension and regular measurement of arterial $\mathrm{CO}_{2}$ tension. The patient re- mained stable during the operation and made an uneventful recovery. Spinal anaesthesia is best avoided in these patients because of a greater risk of hypotension.

Hypocarbia during controlled ventilation in a patient with MoyaMoya disease has been associated with signs of cerebral ischaemic such as seizures, therefore avoidance of hypocarbia and cerebral vasoconstriction is recommended. ${ }^{4}$ In our patient, normocarbia was maintained during the epidural anaesthetic.

We believe that, in the absence of a contraindication, epidural anaesthesia is a better option for Caesarean section in a MoyaMoya patient.

Shiv K. Sharma MB BS MD FRCA

Donald H. Wallace MD

J. Elaine Sidaiwi MD

David R. Gambling MB BS FRCPC

Department of Anesthesiology and Pain Management

University of Texas Southwestern Medical Center

5323 Harry Hines Boulevard

Dallas, TX 75235-9068

\section{REFERENCES}

1 Venkatesh B, Taggart PCM. Anaesthetic management of a patient with moyamoya disease for Caesarean section. Can J Anaesth 1994; 41: 79-80.

2 Ogawa A, Yoshimoto T, Suzuki J, Sakurai Y. Cerebral blood flow in moyamoya disease. Part 1 . Correlation with age and regional distribution. Acta Neurochir (Wien) 1990; 105: 30-4.

3 Konishi Y, Kadowaki C, Hara M, Takeuchi $K$. Aneurysms associated with moyamoya disease. Neurosurgery 1985; 16: 484-91.

4 Bingham RM, Wilkinson DJ. Anaesthetic management of moya-moya disease. Anaesthesia 1985; 40: 1198-202.

5 Hashimoto K, Fujii K, Nishimura K, Kibe M, Kishikawa $T$. Occlusive cerebrovascular disease with moyamoya vessels and intracranial haemorrhage during pregnancy - case report and review of the literature. Neurologia MedicoChirurgica 1988; 28: 588-93.

\section{$R E P L Y$}

We would like to thank Drs. Sharma et al. for their comments on our letter.' We described an anaesthetic technique which resulted in a satisfactory outcome, but did not claim it to be the ideal technique. We discussed the potential hazards of general anaesthesia in this group of patients in our report and took appropriate precautions. We are delighted that the case report by Drs. Sharma et al. provides valuable evidence that regional blocks may also be a safe and suitable method of anaesthesia for Caesarean section in patients with MoyaMoya disease. As this is an uncommon disease, the continued accumulation of evidence for the suitability of various anaesthetic techniques, such as the above, is essential for individualizing anaesthetic management in this group of patients. We would like to emphasize, however, that it is the safe and careful con- 
duct of anaesthesia, rather than the method, which contributes to the successful maternal and fetal outcome.

B. Venkatesh MBBS MD FFARCSI FRCA

University Dept. of Anaesthesia and Intensive Care

Queen Elizabeth Hospital, Edgbastond

Birmingham, B15 2TH

United Kingdom

P.C.M. Taggart MBBS FRCA

Consultant Anaesthetist

George Eliot Hospital, Nuneaton

CV10 7DJ

United Kingdom

REFERENCE

1 Venkatesh B, Taggart PCM. Anaesthetic management of a patient with MoyaMoya disease for Caesarean section (Letter). Can J Anaesth 1994; 41: 79-80.

\section{Anaesthesia for adenotonsillectomy}

To the Editor:

Dr. Webster $e t$ al. are to be commended for examining the role of a prototype armoured laryngeal mask airway for anaesthesia for the adenotonsillectomy in children. ${ }^{1}$ I wish to raise some questions regarding their methods and results and the clinical context of the study.

The authors describe the four anaesthetists taking part in this study as "initially relatively unfamiliar with the LMA for T\&A." They also state that "with practice and the improved conditions obtained when the dose of propofol was increased in each group to $3.5-4.0 \mathrm{mg} \cdot \mathrm{kg}^{-1}$, a satisfactory airway was obtained using the LMA more rapidly with tracheal intubation." Thus, the experimental conditions were changed twice during the study: (1) The anaesthetists became more experienced and (2) the dose of propofol was increased. This may have led to bias against the LMA in the initial phase of the study and may explain "the difficulty in inserting the LMA in ten cases (18.2\%), compared with none during tracheal intubation $(P<0.001)$."

It is not surprising that the heart rate, systolic and mean blood pressure were higher in the ETT group than in the LMA group since all 54 patients in the first group received atropine but only 12 of 54 in the latter group were similarly given atropine. Moreover, the greater blood loss may be attributed to the higher blood pressure. The universal use of atropine in this age group may be a matter of debate, but one can also argue that the healthy children in this study would not have suffered harm if all had received atropine in order to standardize the anaesthetic. In addition, there was no attempt to standardize the surgical technique which may also have introduced bias in the assessment of blood loss.

Similarly, it is not surprising that different results were obtained from the two groups in response to handling of the airway. When the authors calculated the mean duration from $i v$ induction to insertion of the LMA or ETT, did they take into account the extra time required for giving atropine and succinylcholine? In addition, the time to jaw relaxation after propofol can be expected to be shorter than that required for onset of full neuromuscular blockade. The same can be said for the time to return of spontaneous respiration which must have been considerably longer in the ETT group who had been paralyzed. It is misleading to present results as mean and standard deviation when the data are not normally distributed.

Finally, I would like to interpret this study in a clinical context. For the purpose of this study the authors arbitrarily defined desaturation as $\mathrm{SpO}_{2} \leq 94 \%$. What prompted them to pick this number and does transitory desaturation below this point have any clinical relevance? The authors report that the ETT group had lower oxygen saturation on arrival in the recovery room (95.9 \pm 2.21 and $93.5 \pm 4.53, P<0.05$ ). What is the relevance of these numbers when the accuracy of most pulse oximeters is reported to be $\pm 2-3 \% .^{2}$ If the observed difference had caused any clinical concern why were the children not transported to the recovery room with oxygen? The authors quote the work of Patel et al. ${ }^{3}$ who state in their article "The clinical importance of transient saturation of less than $90 \%$ is not known."

The authors' definition of stridor "as a high-pitched laryngeal sound with inspiratory soft tissue and lower rib retraction, but no oxyhaemoglobin desaturation with assisted bag/mask ventilation with $100 \%$ oxygen" hardly deserves to be labelled a complication but rather describes an event which was promptly handled by a vigilant and competent anaesthetist. The same can probably be said for observing brief desaturation with inserting either the LMA or the ETT or with opening the Boyle-Davis gag. It is of note that the more unsettling occurrences of laryngospasm and desaturation $\leq 94 \%$ as reactions to removing the airway were fairly equally distributed between the two groups. Again, what are the clinical consequences of these observations? Did any child need hospitalization subsequent to these events or suffer any damage? Is it not reasonable to state that a certain number of children whose airways are manipulated by a number of different techniques and instruments will respond with breathholding, desaturation or laryngospasm? Observing a variation from a predefined normal state or alteration of a physiological variable does not create a complication per se but serves as a warning signal for a potentially serious 